\title{
Coordinating Flexibility under Uncertainty in Multi-Area AC and DC Grids
}

\author{
Halilbasic, Lejla; Chatzivasileiadis, Spyros; Pinson, Pierre
}

Published in:

Proceedings of 2017 IEEE Manchester PowerTech

Link to article, DOI:

10.1109/PTC.2017.7981034

Publication date:

2017

Document Version

Peer reviewed version

Link back to DTU Orbit

Citation $(A P A)$ :

Halilbasic, L., Chatzivasileiadis, S., \& Pinson, P. (2017). Coordinating Flexibility under Uncertainty in Multi-Area AC and DC Grids. In Proceedings of 2017 IEEE Manchester PowerTech IEEE. https://doi.org/10.1109/PTC.2017.7981034

\section{General rights}

Copyright and moral rights for the publications made accessible in the public portal are retained by the authors and/or other copyright owners and it is a condition of accessing publications that users recognise and abide by the legal requirements associated with these rights.

- Users may download and print one copy of any publication from the public portal for the purpose of private study or research.

- You may not further distribute the material or use it for any profit-making activity or commercial gain

- You may freely distribute the URL identifying the publication in the public portal 


\title{
Coordinating Flexibility under Uncertainty in Multi-Area AC and DC Grids
}

\author{
Lejla Halilbašić, Spyros Chatzivasileiadis, Pierre Pinson \\ Dept. of Electrical Engineering, Technical University of Denmark \\ Kgs. Lyngby, Denmark \\ \{lhal, spchatz,ppin\}@elektro.dtu.dk
}

\begin{abstract}
In the future, mixed AC and DC grids, spanning multiple areas operated by different transmission system operators (TSO), are expected to offer the necessary controllability for integrating large amounts of intermittent renewable generation. This is facilitated by high voltage direct current transmission based on voltage source converter technology that can offer recourse actions in the form of preventive and corrective control of both active and reactive power. Market-clearing procedures, based on optimal power flow algorithms, need to be revised to account for DC transmission, flexibility and privacy requirements. To this end, we propose a decentralized two-stage stochastic market-clearing algorithm that incorporates meshed DC grids and allows the sharing of flexibility resources between areas. The benefit of this approach lies in its pricing mechanism, used for coordinating the different area subproblems and requiring only a moderate exchange of information while ensuring systemwide optimality. Case studies are presented to illustrate the methodology and to demonstrated the benefits of additional controllability provided by DC grids.

Index Terms-Decomposition, multi-area optimal power flow, multi-terminal HVDC, stochastic programming.
\end{abstract}

\section{NOMENCLATURE}

\section{A. Indices and sets}

$G \quad$ Set of generators.

$D \quad$ Set of demand.

$N \quad$ Set of nodes.

$S \quad$ Set of wind power scenarios.

$\Gamma \quad$ Set of external lines (i.e. tie-lines).

$A \quad$ Area index.

$A A$ Index for neighboring areas.

$A C$ Index for terms belonging to AC grid.

$D C$ Index for terms belonging to DC grid.

$c \quad$ Converter index.

$g \quad$ Generator index.

$d \quad$ Load/demand index.

$l \quad$ Line index.

$n, m$ Node indices.

$s \quad$ Scenario index.

$v \quad$ Iteration index.

$w \quad$ Wind generator index.

\section{B. Parameters}

$c_{g} \quad$ Generator cost function.

This work is supported by the European project BEST PATHS under the 7th Framework Programme, Grant No. 612748.

\begin{tabular}{|c|c|}
\hline$c_{g}^{R U}$ & $\begin{array}{l}\text { Cost of unit } g \text { for providing up-reserve capacity } \\
\text { [€/MWh]. }\end{array}$ \\
\hline$c_{g}^{R D}$ & $\begin{array}{l}\text { Cost of unit } g \text { for providing down-reserve capacity } \\
\text { [€/MWh]. }\end{array}$ \\
\hline $\mathbf{I}$ & Incidence matrix [-]. \\
\hline $\mathbf{p}_{\mathrm{ws}}$ & $\begin{array}{l}\text { Actual wind power production in scenario } s \\
{[\mathrm{MW}] \text {. }}\end{array}$ \\
\hline$r_{l}$ & Resistance of DC line $l$ [p.u.]. \\
\hline & Reactance of AC line $l$ [p.u.]. \\
\hline$V^{L O L}$ & Value of lost load [€/MWh]. \\
\hline$\pi_{s}$ & Probability of scenario $s[-]$. \\
\hline \multicolumn{2}{|c|}{ C. Variables } \\
\hline $\mathbf{F}_{1}$ & Day-ahead flow on intra-area lines [MW]. \\
\hline $\mathbf{P}_{\mathbf{c}}$ & Day-ahead converter power [MW]. \\
\hline $\mathbf{P}_{\mathrm{g}}$ & Day-ahead conventional generation [MW]. \\
\hline $\mathbf{P}_{\mathbf{d}}$ & Demand [MW]. \\
\hline $\mathbf{P}_{\mathbf{w}}$ & Day-ahead wind power production [MW]. \\
\hline $\mathbf{T}_{1}$ & Day-ahead flow on tie-lines [MW]. \\
\hline$U_{n}$ & Day-ahead voltage magnitude at DC node $n$ [p.u.]. \\
\hline$\delta_{n}$ & Day-ahead voltage angle at AC node $n$ [rad]. \\
\hline $\mathbf{f}_{\mathbf{l s}}$ & Balancing flow on intra-area lines during $s[\mathrm{MW}]$. \\
\hline & Balancing converter power during $s$ [MW]. \\
\hline$p_{d s}^{\text {shed }}$ & Balancing converter power during $s[\mathrm{MW}]$. \\
\hline $\mathbf{p}_{\mathrm{ws}}^{\mathrm{spill}}$ & Wind spillage during $s[\mathrm{MW}]$. \\
\hline$r_{g s}^{U}$ & Deployed up-reserve capacity during $s$ [MW]. \\
\hline$r_{g s}^{D}$ & Deployed down-reserve capacity during $s[\mathrm{MW}]$. \\
\hline$t_{l s}$ & Balancing flow on tie-lines during $s[\mathrm{MW}]$. \\
\hline$u_{n s}$ & $\begin{array}{l}\text { Balancing voltage magnitude at DC node } n \text { during } s \\
\text { [p.u.]. }\end{array}$ \\
\hline$\theta_{n s}$ & Balancing voltage angle at AC node $n$ during $s$ [rad]. \\
\hline$\mu_{l}$ & Day-ahead power export price on tie-line $l$ [€/MWh]. \\
\hline$\nu_{l s}$ & $\begin{array}{l}\text { Balancing power export price on tie-line } l \text { during } s \\
\text { [€/MWh]. }\end{array}$ \\
\hline
\end{tabular}

\section{INTRODUCTION}

The large scale integration of renewable energy sources (RES) has introduced significant uncertainties in power system planning and operation. This calls for flexibility and market setups that explicitly account for fluctuating and partially predictable generation [1]. In the future, in Europe, China, and the US, mixed AC and DC grids, spanning multiple areas controlled by different transmission system operators 
(TSO), are expected to offer the necessary controllability for integrating large amounts of RES. High voltage direct current transmission based on voltage source converter technology (VSC-HVDC) has significant benefits compared to AC and conventional HVDC technology. It can offer recourse actions, in the form of preventive and corrective control, and enables the formation of meshed HVDC grids which are very likely to evolve from already existing point-to-point connections [2].

To this end, optimal power flow algorithms (OPF), used for clearing electricity markets, need to be revised in order to (a) optimally coordinate mixed AC and DC grids in a multi-area setting, (b) avoid the disclosure of sensitive intra-area data, (c) incorporate recourse actions offered by DC grids and (d) provide a market for reserve capacity. Ideally, this coordination would be done by a central entity with access to all data, but the restriction imposed by (b) calls for methods which decompose the OPF by area. This decentralization can be done by decomposition techniques that limit the amount of information shared between areas while ensuring system-wide optimality. In [3] an OPF decomposition is performed using Augmented Lagrangian Relaxation with multiplier updates based on the Auxiliary Problem Principle, whereas [4] decomposes the problem using Lagrangian Relaxation (LR) combined with a subgradient method. Both methods have the drawback of using multiplier updates whose convergence behavior is determined by parameters that must be tuned depending on the system. In [5] a multi-area OPF is solved using chance-constraints to account for wind power forecast errors. However, this setup only considers point-to-point HVDC interconnections and does not account for an explicit reserve capacity market that incentivizes the provision of regulating power.

To complement these findings, we present a decentralized two-stage stochastic market-clearing algorithm that incorporates meshed DC grids and their flexibility while ensuring data privacy of each regional TSO. Flexibility resources are shared between areas and priced by a flexibility market. In contrast to the decomposition methods applied in [3], [4], we choose Optimality Condition Decomposition (OCD), a LR based method, which does not need any parameter tuning or central coordination [6], [7]. Prices used for coordinating the different area subproblems, do not need to be estimated with auxiliary update methods, that impact the performance of decomposition methods. Prices on power exports along tielines are provided by each area and can be used as costs in neighboring areas objective functions. This allows for a fully decentralized solution, but comes at a cost of requiring a higher information exchange, where primal and dual variables are exchanged. A reduced number of exchanged variables would enable a distributed solution but would require a coordinating entity. Reserve capacity markets are accounted for and make preventive and corrective control actions available across the entire system alleviating uncertainties arising from RES.

The remainder of this paper is organized as follows. Section II describes the mathematical formulation of the centralized and decentralized market-clearing models. II-B explains the decomposition and the iterative algorithm. In Section III the methodology is applied to a modified version of the IEEE Two Area RTS-96 test system. A case study is presented that shows the impact of additional controllability due to the DC grid in Section III-B. In Section III-C we test the algorithm on a three area system to verify its adaptability to systems with more than two areas. Section IV concludes.

\section{MATHEMATICAL FORMULATION}

The mathematical formulation of the centralized multiarea OPF and its equivalent decomposed formulation are given in the following section. The market-clearing model is formulated as a two-stage stochastic program optimizing the energy and reserve dispatch simultaneously [8]. The only source of uncertainty considered is wind power represented by a finite set of scenarios. The market is cleared in a singleperiod auction omitting inter-temporal constraints, such as ramping limits. As usual in market applications, the network is modeled using a linear DC approximation. Conventional generators and loads are connected to $\mathrm{AC}$ nodes only. Wind farms are assumed to be located at AC and DC nodes.

\section{A. Centralized OPF Formulation}

The centralized DC-OPF for the entire power system is formulated below.

1) Objective Function: The objective function to be minimized represents day-ahead and balancing costs in expectation:

$$
\begin{aligned}
\min _{\Xi} & \sum_{A}\left\{\sum_{g \in G^{A}}\left[c_{g}\left(P_{g}\right)+c_{g}^{R U} R_{g}^{U}+c_{g}^{R D} R_{g}^{D}\right]\right. \\
& +\sum_{s \in S} \pi_{s}\left(\sum_{g \in G^{A}}\left[c_{g}\left(P_{g}+r_{g s}^{U}-r_{g s}^{D}\right)\right]\right. \\
& \left.\left.+\sum_{d \in D^{A}} V^{L O L} p_{d s}^{s h e d}\right)\right\}
\end{aligned}
$$

where $\Xi$ is the set of first- and second-stage decision variables. Second-stage decisions dependent on particular scenario realizations are indicated with subscript $s$.

Reserve capacity costs $c_{g}^{R U}$ and $c_{g}^{R D}$ are incorporated in the form of reserve capacity bids. Recourse actions $\mathbf{r}_{\mathbf{s}}$ include up- and down-reserve deployment $\left(r_{g s}^{U}\right.$ and $\left.r_{g s}^{D}\right)$, load shedding $p_{d s}^{s h e d}$, wind spillage $p_{w s}^{s p i l l}$, converter setpoint changes $p_{c s}$ and thus power flows on intra- and inter-area DC links, denoted with $f_{l s}^{D C}$ and $t_{l s}^{D C}$, respectively. Out of these, costs are assigned to load shedding, in the form of penalties, and reserve deployment, which correspond to energy production costs. Other recourse actions are not penalized in the objective function.

2) Equality Constraints: The set of equality constraints comprises power balance and line flow equations for the day-ahead and real-time stage, respectively. Power balance equations are formulated in (2) to (5):

$$
\begin{aligned}
\mathbf{I}_{\mathbf{n g}} \mathbf{P}_{\mathbf{g}} & +\mathbf{I}_{\mathbf{n w}} \mathbf{P}_{\mathbf{w}}-\mathbf{I}_{\mathbf{n c}} \mathbf{P}_{\mathbf{c}} \\
& -\mathbf{I}_{\mathbf{n l}} \mathbf{F}_{\mathbf{l}}-\mathbf{I}_{\mathbf{n l}} \mathbf{T}_{\mathbf{l}}-\mathbf{I}_{\mathbf{n d}} \mathbf{P}_{\mathbf{d}}=0, \quad \forall n \in N^{A C}
\end{aligned}
$$




$$
\begin{gathered}
\mathbf{I}_{\mathbf{n w}} \mathbf{P}_{\mathbf{w}}+\mathbf{I}_{\mathbf{n c}} \mathbf{P}_{\mathbf{c}}-\mathbf{I}_{\mathbf{n l}} \mathbf{F}_{\mathbf{l}}-\mathbf{I}_{\mathbf{n l}} \mathbf{T}_{\mathbf{l}}=0, \quad \forall n \in N^{D C} \\
\mathbf{I}_{\mathbf{n g}}\left(\mathbf{r}_{\mathbf{g s}}^{\mathbf{U}}-\mathbf{r}_{\mathbf{g s}}^{\mathbf{D}}\right)+\mathbf{I}_{\mathbf{n w}}\left(\mathbf{p}_{\mathbf{w s}}-\mathbf{p}_{\mathbf{w s}}^{\text {spill }}-\mathbf{P}_{\mathbf{w}}\right) \\
-\mathbf{I}_{\mathbf{n c}}\left(\mathbf{p}_{\mathbf{c s}}-\mathbf{P}_{\mathbf{c}}\right)-\mathbf{I}_{\mathbf{n l}}\left(\mathbf{f}_{\mathbf{l s}}-\mathbf{F}_{\mathbf{l}}\right)-\mathbf{I}_{\mathbf{n l}}\left(\mathbf{t}_{\mathbf{l s}}-\mathbf{T}_{\mathbf{l}}\right) \\
\quad-\mathbf{I}_{\mathbf{n d}} \mathbf{p}_{\mathbf{d s}}^{\text {shed }}=0, \quad \forall n \in N^{A C}, \forall s \\
\mathbf{I}_{\mathbf{n w}}\left(\mathbf{p}_{\mathbf{w s}}-\mathbf{p}_{\mathbf{w s}}^{\text {spill }}-\mathbf{P}_{\mathbf{w}}\right)+\mathbf{I}_{\mathbf{n c}}\left(\mathbf{p}_{\mathbf{c s}}-\mathbf{P}_{\mathbf{c}}\right) \\
-\mathbf{I}_{\mathbf{n l}}\left(\mathbf{f}_{\mathbf{l s}}-\mathbf{F}_{\mathbf{l}}\right)-\mathbf{I}_{\mathbf{n l}}\left(\mathbf{t}_{\mathbf{l s}}-\mathbf{T}_{\mathbf{l}}\right)=0, \quad \forall n \in N^{D C}, \forall s
\end{gathered}
$$

Matrices and vectors are indicated with bold symbols. Dayahead power balance equations of the AC (2) and DC grid (3) are coupled through controllable converter power flows $\mathbf{P}_{\mathbf{c}}$. Real-time power balance constraints (4) and (5) link recourse actions to day-ahead decisions depending on the actual wind power realization $\mathbf{p}_{\mathrm{ws}}$. Incidence matrices are denoted with $\mathbf{I}$ and indicate the location of generators, wind farms, converters, lines and loads.

Power flows in the DC grid are modeled according to [9]. $\mathrm{AC}$ and $\mathrm{DC}$ tie-line flow equations are given by:

$$
\begin{gathered}
T_{l}=\frac{\delta_{n}-\delta_{m}}{x_{l}}: \mu_{l}^{A C}, \quad \forall l=(n, m) \in \Gamma^{A C} \\
T_{l}=\frac{U_{n}-U_{m}}{r_{l}}: \mu_{l}^{D C}, \quad \forall l=(n, m) \in \Gamma^{D C} \\
t_{l s}=\frac{\theta_{n s}-\theta_{m s}}{x_{l}}: \nu_{l s}^{A C}, \quad \forall l=(n, m) \in \Gamma^{A C}, \forall s \\
t_{l s}=\frac{u_{n s}-u_{m s}}{r_{l}}: \nu_{l s}^{D C}, \quad \forall l=(n, m) \in \Gamma^{D C}, \forall s .
\end{gathered}
$$

Dual variables associated with tie-line flow constraints are indicated after the colon. Intra-area AC and DC line flows, $F_{l}$, are modeled in the same way and are not explicitly mentioned here. The voltage angle of the AC slack bus is set to zero.

3) Inequality Constraints: Day-ahead and real-time inequality constraints comprise line capacity, converter power, voltage, generation and reserve capacity limits. Minimum and maximum voltage levels of DC buses are set to 0.9 and 1.1 p.u., respectively.

\section{B. Decentralized OPF Formulation}

$\mathrm{AC}$ and DC tie-line flow constraints (6) to (9) contain variables from more than one area. They constitute complicating constraints and prevent the OPF of the interconnected power system to be decomposed per area. To tackle this, we use the Optimality Condition Decomposition (OCD) technique, an iterative solution procedure, which is an extension to the common LR method and based on the decoupling of the first-order Karush-Kuhn-Tucker (KKT) conditions. The KKT conditions of the original problem correspond to the superposition of all subproblems' KKT conditions. A more detailed analysis of the method and conditions for convergence are provided in [10].

As opposed to LR, where all complicating constraints of the entire system are augmented as soft constraints to each area's objective function, OCD only augments complicating constraints belonging to neighboring areas (indicated with $A A$ in equations (10) to (14)) [11]. As a result, each area can keep its own complicating constraints in the constraint set by treating neighboring areas' decision variables as parameters and setting them to the values obtained in the previous iteration. The benefit of this approach is, that dual variables associated with tie-line flow constraints and used as cost parameters when augmenting the constraints to neighboring areas' objective functions, are readily available and do not need to be estimated with auxiliary methods, such as ADMM. Decomposition methods dependent on auxiliary update methods are prone to divergence, if their parameters are not properly tuned. OCD allows for a fully decentralized solution, given that all computation is done within the scopes of the subproblems. Hence, the need for a central entity is avoided and a coordinated solution is achieved by exchanging only a moderate amount of information.

Each area's OPF $(A)$ is formulated as follows:

$$
\begin{aligned}
\min _{\Xi A} & C^{D, A}+\mathbb{E}\left[C_{s}^{B, A}\right] \\
& +\sum_{l \in \Gamma^{A C, A}} \hat{\mu}_{l}^{A C, A A}\left(\frac{\hat{\delta}_{m}^{A A}-\delta_{n}^{A}}{x_{l}}-\hat{T}_{l}^{A A}\right) \\
& +\sum_{l \in \Gamma^{D C, A}} \hat{\mu}_{l}^{D C, A A}\left(\frac{\hat{U}_{m}^{A A}-U_{n}^{A}}{x_{l}}-\hat{T}_{l}^{A A}\right) \\
& +\sum_{s}\left\{\sum_{l \in \Gamma^{A C, A}} \hat{\nu}_{l s}^{A C, A A}\left(\frac{\hat{\theta}_{m s}^{A A}-\theta_{n s}^{A}}{x_{l}}-\hat{t}_{l s}^{A A}\right)\right. \\
& \left.+\sum_{l \in \Gamma^{D C, A}} \hat{\nu}_{l s}^{D C, A A}\left(\frac{\hat{u}_{m s}^{A A}-u_{n s}^{A}}{r_{l}}-\hat{t}_{l s}^{A A}\right)\right\}
\end{aligned}
$$

s.t. Constraints in II-A2 for area A

$$
\text { Constraints in II-A3 for area A }
$$

$$
\begin{aligned}
& T_{l}^{A}=\frac{\delta_{n}^{A}-\hat{\delta}_{m}^{A A}}{x_{l}}: \mu_{l}^{A C, A}, \forall l=(n, m) \in \Gamma^{A C} \\
& T_{l}^{A}=\frac{U_{n}^{A}-\hat{U}_{m}^{A A}}{r_{l}}: \mu_{l}^{D C, A}, \forall l=(n, m) \in \Gamma^{D C} \\
& t_{l s}^{A}=\frac{\theta_{n s}^{A}-\hat{\theta}_{m s}^{A A}}{x_{l}}: \nu_{l s}^{A C, A}, \forall l=(n, m) \in \Gamma^{A C}, \forall s \\
& t_{l s}^{A}=\frac{u_{n s}^{A}-\hat{u}_{m s}^{A A}}{r_{l}}: \nu_{l s}^{D C, A}, \forall l=(n, m) \in \Gamma^{D C}, \forall s
\end{aligned}
$$

$C^{D, A}$ and $C_{s}^{B, A}$ represent area $A$ 's day-ahead and balancing costs, respectively. Hatted terms are constants with values obtained from the neighboring areas' most recent iteration. Note that balancing tie-line flow constraints (i.e., stochastic complicating constraints) do not need to be weighted by their probabilities when augmented to the objective function (10), since dual variables of stochastic constraints in the primal problem already contain information on their probability of occurrence. After each iteration border node state variables (i.e. voltage angles for $\mathrm{AC}$ tie-lines and voltage magnitudes for DC tie-lines) and dual variables associated with tie-line 
flow constraints are exchanged. The dual variables exchanged represent prices on power imports/exports along tie-lines. The algorithm is suitable for both sequential and parallel implementation.

For systems with a low number of primal consensus variables, which connected areas need to agree on, tie-line flow mismatches can be used as convergence criteria. In this case, the algorithm converges as soon as all tie-line flow mismatches are within tolerance:

$$
\begin{gathered}
\left|T_{l}^{A}+T_{l}^{A A}\right| \leq \epsilon, \quad \forall l \in \Gamma^{A C} \cup \Gamma^{D C} \\
\left|t_{l s}^{A}+t_{l s}^{A A}\right| \leq \epsilon, \quad \forall l \in \Gamma^{A C} \cup \Gamma^{D C}, \forall s .
\end{gathered}
$$

A large number of primal consensus variables significantly increases computation time. Instead, convergence criteria based on dual variables, which tend to converge faster, can be used. In this case, the algorithm converges, if dual variables do not change significantly in two consecutive iterations:

$$
\begin{gathered}
\left|\mu_{l}^{v}-\mu_{l}^{v-1}\right| \leq \epsilon, \quad \forall l \in \Gamma^{A C} \cup \Gamma^{D C} \\
\mathbb{E}\left[\left|\nu_{l s}^{v}-\nu_{l s}^{v-1}\right|\right] \leq \epsilon, \quad \forall l \in \Gamma^{A C} \cup \Gamma^{D C} .
\end{gathered}
$$

Equation (17) refers to the change in dual variables of the dayahead tie-line flow constraints, whereas criterion (18) checks for the expected change in balancing tie-line flow prices. Thus, convergence is evaluated on the basis of $2 \times|\Gamma|$ criteria, where $|\Gamma|$ denotes the cardinality of the total tie-line set.

\section{CASE STUdy}

All simulations were carried out in Python using the Gurobi Optimizer [12]. The Python code will be made available online.

\section{A. IEEE Two Area RTS-96 test system with six-node DC} overlay grid

The algorithm is tested on a modified version of the IEEE Two Area RTS-96 system [13] with an additional six-node DC overlay grid. The system is depicted in Fig. 1 with the DC grid highlighted in purple. The two areas are connected with two $\mathrm{AC}$ tie-lines and one DC tie-line. Each area contains three DC nodes, three internal DC lines, 12 conventional generators and six wind farms of 200 MW capacity each. AC tie-line parameters correspond to the ones of the original IEEE Two Area RTS-96 test system [14]. All DC lines have a resistance of 0.002 p.u. and a capacity of $300 \mathrm{MW}$. Power ratings of $500 \mathrm{MW}$ are chosen for all converters. We consider a high demand snapshot with a total system load of $4770.9 \mathrm{MW}$ for the single-period auction. Day-ahead wind power generation is set to its expected value. A set of 100 equally probable wind generation scenarios is used, which has been derived from wind measurement data of a location in western Denmark and made available online [15], [16]. The value of lost load $V^{L O L}$ is chosen with $1000 € / \mathrm{MWh}$. The dual convergence criteria stated in equations (17) and (18) are chosen with a tolerance $\epsilon$ of $0.01 €$. Quadratic cost functions are assumed for conventional generators. The area splitting is performed along geographical borders. TSOs are responsible for the operation

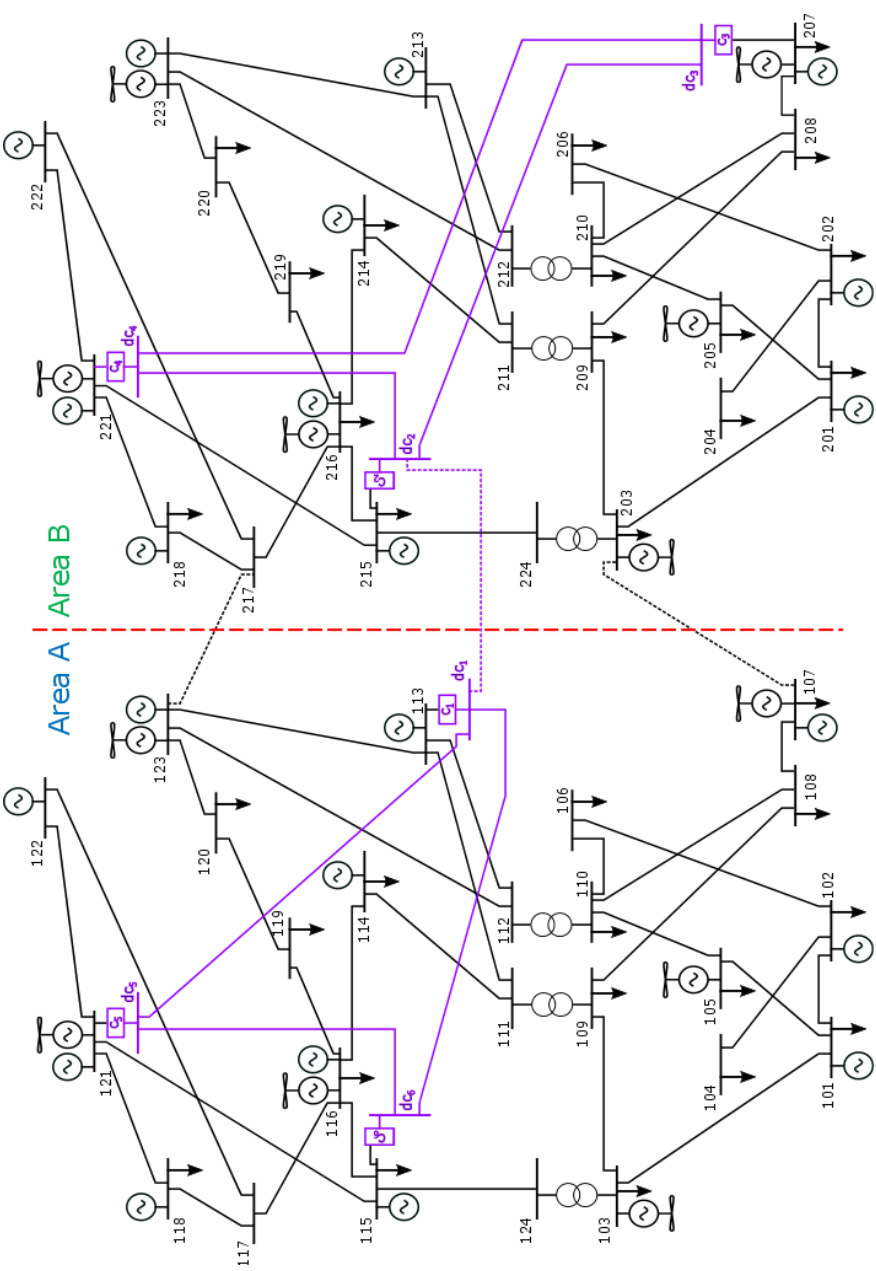

Fig. 1. Two-area IEEE RTS96 test system with six-node DC overlay grid. Tie-lines are illustrated with dashed lines.

of those parts of the interconnected AC and DC system that are located within their jurisdiction. The subproblems are solved sequentially. Alternatively, the algorithm is suitable for parallel implementation.

The decentralized algorithm converges after 19 iterations for the chosen tolerance. The results are listed in Table I. The cost difference between the centralized and the decentralized solution amounts to $0.04 \%$. Fig. 2 depicts the convergence of the total system cost compared to the optimal centralized solution. The convergence behavior of the balancing tie-line flow prices for a particular scenario is illustrated in Fig. 3. It can be observed that the convergence behavior does not suffer from fluctuations as often seen in iterative solution procedures. Oscillations are quickly damped due to the availability of dual variables, which do not need to be estimated with auxiliary methods that determine the performance of the algorithm depending on the choice of parameters. Actual tie-line flow mismatches are below $1 \mathrm{MW}$ for the day-ahead stage and at most $2.05 \mathrm{MW}$ for the balancing stage in expectation, as shown in Table II. If necessary, tie-line flows would have to be adjusted by the two TSOs in agreement with each 
other to offset the minor mismatches. The price of avoiding the disclosure of sensitive intra-area data is the higher computation time of the decentralized case compared to the centralized solution. However, improvements can be achieved with a parallel implementation and by performing only single Newton-Raphson steps instead of solving the subproblems until optimality at each iteration. Nevertheless, it should be noted that this work focuses mainly on methods to preserve each area's independence, rather than increasing computational efficiency.

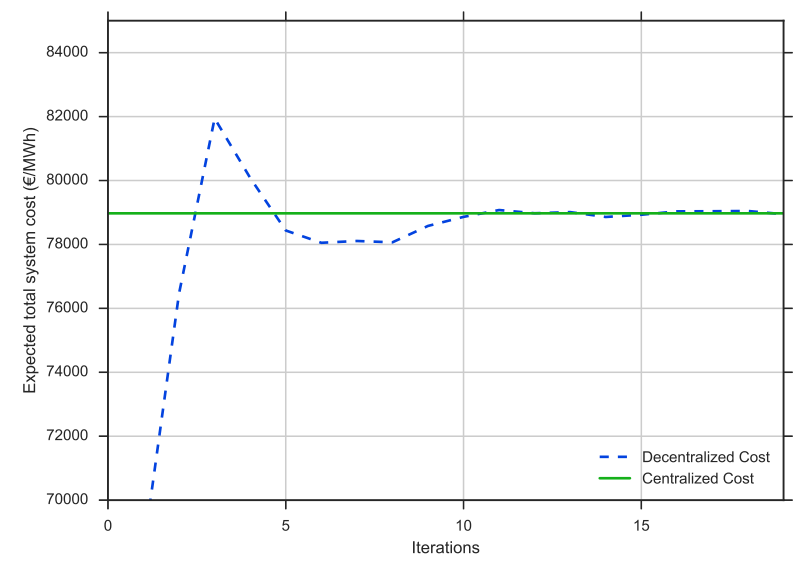

Fig. 2. Convergence of expected total system cost for a single-period auction.

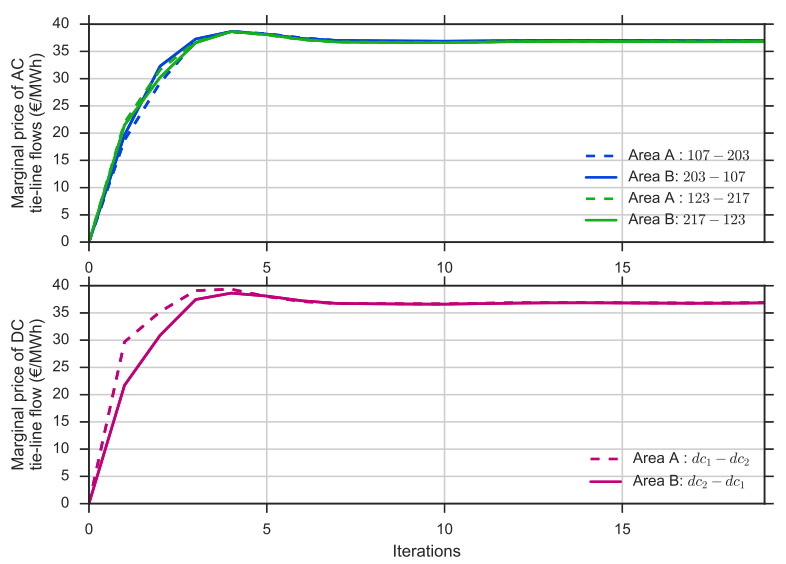

Fig. 3. Convergence of marginal prices of balancing tie-line flow constraints for scenario 60

\section{B. IEEE Two Area RTS-96 test system: HVAC vs. HVDC}

To demonstrate the impact of increased controllability due to the DC grid, the overlay DC grid of the system used in Section III-A is replaced with AC transmission lines. Thus, the set of available recourse actions is reduced to reserve deployment, wind spillage and load shedding, and does not include converter setpoint changes and DC line flows. The line capacities of the overlay HVAC grid are set to 300
TABLE I

RESULTS FOR THE TWO AREA TEST SYSTEM WITH HVDC OVERLAY GRID.

\begin{tabular}{lcccc}
\hline Model & $\begin{array}{c}\text { Total Cost } \\
(€ / \mathrm{h})\end{array}$ & $\begin{array}{c}\text { Number of } \\
\text { iterations } v\end{array}$ & $\begin{array}{c}\text { Cost difference } \\
(\%)\end{array}$ & $\begin{array}{c}\text { Runtime } \\
(\mathrm{s})\end{array}$ \\
\hline Centralized & 78973.51 & - & 0.04 & 1.71 \\
Decentralized & 78945.69 & 19 & & 27.07 \\
\hline
\end{tabular}

TABLE II

TIE-LINE FLOW MISMATCHES FOR THE TWO AREA TEST SYSTEM WITH A DC OVERLAY GRID.

\begin{tabular}{ccc}
\hline $\begin{array}{c}\text { Tie-line } l \\
(-)\end{array}$ & $\begin{array}{c}\text { Day-ahead } \\
\text { (MW) }\end{array}$ & $\begin{array}{c}\text { Balancing } \\
\text { (MW) }\end{array}$ \\
\hline $107-203$ & 0.02 & 0.72 \\
$123-217$ & 0.67 & 2.05 \\
$d c_{1}-d c_{2}$ & 0.71 & 0.05 \\
\hline
\end{tabular}

MW, corresponding to the values of the DC lines used in the previous section. The reactances of all new AC lines are chosen with 0.0628 p.u.. Note that buses 115 and 121 as well as 215 and 221 are already connected through $\mathrm{AC}$ lines in the original system. Their line parameters are adjusted to account for the new AC lines inserted in parallel.

Table III lists the results for both HVAC and HVDC system configurations. The total system cost is higher for the HVAC case. Even though less reserve capacity is scheduled, dayahead costs are higher, which indicates that more expensive generators need to be deployed for generation and reserve provision. In the HVDC case, more down-reserve capacity is scheduled allowing for more wind power to be accommodated by the system in scenarios with wind power surplus. This also leads to a lower amount of expected wind spillage resulting in cost savings due to the replacement of more expensive conventional generation by wind power. The DC grid for the case studies presented in this paper has not been chosen optimally and serves only the demonstration of the decentralized market-clearing algorithm. Thus, there is still room for improvement in the cost savings from DC grids, whose benefits are not fully captured here. As shown in table $\mathrm{IV}$, the decentralized algorithm for the HVAC case converges after 20 iterations to a solution with a deviation of $0.02 \%$ from the centralized system cost.

TABLE III

COMPARISON OF HVAC AND HVDC OVERLAY GRID.

\begin{tabular}{lcc}
\hline & HVAC & HVDC \\
\hline Total System Cost $(€ / \mathrm{h})$ & 79160.03 & 78973.5 \\
Day-ahead System Cost $(€ / \mathrm{h})$ & 73275.2 & 73199.5 \\
Total up-reserve capacity $R^{U}(\mathrm{MW})$ & 1100 & 1100 \\
Total down-reserve capacity $R^{D}(\mathrm{MW})$ & 202.3 & 209.4 \\
Total expected wind spillage $(\mathrm{MW})$ & 32.7 & 30.2
\end{tabular}


TABLE IV

RESULTS FOR TWO AREA TEST SYSTEM WITH HVAC GRID.

\begin{tabular}{lcccc}
\hline Model & $\begin{array}{c}\text { Total Cost } \\
(€ / \mathrm{h})\end{array}$ & $\begin{array}{c}\text { Number of } \\
\text { iterations } v\end{array}$ & $\begin{array}{c}\text { Cost difference } \\
(\%)\end{array}$ & $\begin{array}{c}\text { Runtime } \\
(\mathrm{s})\end{array}$ \\
\hline Centralized & 79160.03 & - & 0.02 & 1.51 \\
Decentralized & 79174.64 & 20 & & 22.38 \\
\hline
\end{tabular}

TABLE V

RESULTS FOR THREE AREA TEST SYSTEM.

\begin{tabular}{lccc}
\hline Model & $\begin{array}{c}\text { Total Cost } \\
(€ / \mathrm{h})\end{array}$ & $\begin{array}{c}\text { Number of } \\
\text { iterations } v\end{array}$ & $\begin{array}{c}\text { Cost difference } \\
(\%)\end{array}$ \\
\hline Centralized & 120343.25 & - & 0.34 \\
Decentralized & 120753.87 & 27 & \\
\hline
\end{tabular}

\section{IEEE Three Area RTS-96 test system with seven-node DC overlay grid}

In order to verify the adaptability of the algorithm to more than two areas, we have applied the method to a modified version of the IEEE Three Area RTS-96 test system. The two areas $A$ and $B$ of the system depicted in Fig. 1 are identical regarding their AC grids. A third area $C$ with the same internal $\mathrm{AC}$ structure has been added to the system. A new DC node, $d c_{7}$, is added to $C$ and connected through a converter to the AC node 318 . The set of inter-area lines is extended to include one AC tie-line between $B$ and $C$ (i.e., from node 223 to node 317 ) and one DC tie-line between $A$ an $C$ (i.e., from node $d c_{5}$ to $d c_{7}$ ). The parameters of the new $\mathrm{AC}$ tie-line correspond to the ones of the original IEEE Three Area test system [14], while the parameters for the new DC tie-line have been chosen as in Section III-A. The dual convergence criteria stated in equations (17) and (18) are chosen with $\epsilon$ equal to $0.01 €$.

As shown in table $\mathrm{V}$, the algorithm converges after 27 iterations. The difference between the centralized and the decentralized solution amounts to $0.34 \%$. This shows that the algorithm can be generalized to cases with more than two areas.

\section{CONClusion And Future Work}

An iterative method to decentralize the market-clearing of mixed AC and DC grids by area has been presented. Flexibility resources are made available across the entire system and are more easily accessible due to the additional controllability provided by DC grids. Fast convergence is achieved while requiring only a moderate exchange of information between neighbors preserving each area's independence. The method has proven to be scalable and thus, applicable to large systems that cannot be solved centrally. In large networks with several areas we expect the computation of the decentralized solution to be equally good or even faster than in the centralized case.

It has been shown in [17] that the performance of OCD largely depends on the splitting and coupling between areas.
Future work will investigate how to optimally split large interconnected systems comprising AC and DC grids, while allowing for an efficient decentralized solution. This could also give an indication on the operation of a future DC grid, whether it should be integrated in or decoupled from current AC grid operations. The algorithm presented in this paper could provide a framework for DC grid operators to offer preventive and corrective control as services to the $\mathrm{AC}$ grid using the pricing mechanism presented. Alternative ways to incorporate uncertainties and recourse actions in OCD algorithms will be studied.

\section{REFERENCES}

[1] S. Delikaraoglou, P. Pinson, R. Eriksson, and T. Weckesser, "Optimal Dynamic Capacity Allocation of HVDC Interconnections for Crossborder Exchange of Balancing Services in Presence of Uncertainty Extended Version," ArXiv e-prints, Feb. 2015, arXiv:1503.00195.

[2] http://www.bestpaths-project.eu/.

[3] B. H. Kim and R. Baldick, "Coarse-Grained Distributed Optimal Power Flow," IEEE Transactions on Power Systems, vol. 12, no. 2, pp. 932939, May 1997.

[4] A. J. Conejo and J. A. Aguado, "Multi-Area Coordinated Decentralized DC Optimal Power Flow," IEEE Transactions on Power Systems, vol. 13 , no. 4, pp. 1272-1278, Nov 1998.

[5] M. Vrakopoulou, S. Chatzivasileiadis, E. Iggland, M. Imhof, T. Krause, O. Makela, J. Mathieu, L. Roald, R. Wiget, and G. Andersson, "A Unified Analysis of Security-Constrained OPF Formulations Considering Uncertainty, Risk, and Controllability in Single and Multi-Area Systems," in 2013 IREP Symposium, Rethymnon, Greece, Aug 2013.

[6] F. Nogales, F. Prieto, and A. Conejo, "A Decomposition Methodology Applied to the Multi-Area Optimal Power Flow Problem," Annals of Operations Research, vol. 120, no. 1, pp. 99-116, 2003.

[7] G. Hug-Glanzmann and G. Andersson, "Decentralized Optimal Power Flow Control for Overlapping Areas in Power Systems," IEEE Transactions on Power Systems, vol. 24, no. 1, pp. 327-336, Feb 2009.

[8] J. M. Morales, A. J. Conejo, H. Madsen, P. Pinson, and M. Zugno, Integrating Renewables in Electricity Markets: Operational Problems. Springer, 2014.

[9] R. Wiget and G. Andersson, "DC Optimal Power Flow Including HVDC Grids," in Electrical Power Energy Conference (EPEC), 2013 IEEE, Aug 2013, pp. 1-6.

[10] A. J. Conejo, F. J. Nogales, and F. J. Prieto, "A Decomposition Procedure based on Approximate Newton Directions," Mathematical Programming, vol. 93, no. 3, pp. 495-515, 2002.

[11] A. Ahmadi-Khatir, A. J. Conejo, and R. Cherkaoui, "Multi-Area Energy and Reserve Dispatch Under Wind Uncertainty and Equipment Failures," IEEE Transactions on Power Systems, vol. 28, no. 4, pp. 4373-4383, Nov 2013.

[12] Gurobi Optimization, "Gurobi Optimizer Reference Manual," 2015. [Online]. Available: http://www.gurobi.com

[13] C. Ordoudis, P. Pinson, J. M. Morales, and M. Zugno, An Updated Version of the IEEE RTS 24-Bus System for Electricity Market and Power System Operation Studies. Technical University of Denmark, 2016.

[14] C. Grigg, P. Wong, P. Albrecht, R. Allan, M. Bhavaraju, R. Billinton, Q. Chen, C. Fong, S. Haddad, S. Kuruganty, W. Li, R. Mukerji, D. Patton, N. Rau, D. Reppen, A. Schneider, M. Shahidehpour, and C. Singh, "The IEEE Reliability Test System-1996. A Report Prepared by the Reliability Test System Task Force of the Application of Probability Methods Subcommittee," IEEE Transactions on Power Systems, vol. 14, no. 3, pp. 1010-1020, Aug 1999.

[15] W. A. Bukhsh, C. Zhang, and P. Pinson, "An Integrated Multiperiod OPF Model With Demand Response and Renewable Generation Uncertainty," IEEE Transactions on Smart Grid, vol. 7, no. 3, pp. 1495-1503, May 2016.

[16] P. Pinson, "Wind Energy: Forecasting Challenges For Its Operational Management," Statist. Sci, pp. 564-585, 2013.

[17] J. Guo, G. Hug, and O. Tonguz, "Impact of Partitioning on the Performance of Decomposition Methods for AC Optimal Power Flow," in 2015 IEEE Power Energy Society Innovative Smart Grid Technologies Conference (ISGT), Feb 2015, pp. 1-5. 